\title{
AMOSTRAGEM DOMICILIAR CONTINUA EM ESTUDOS EPIDEMIOLÓGICOS E NO ENSINO *
}

José da Rocha Carvalheiro** Odécio Sanches ***

Carvalheiro, J. DA R. \& SANChES, O. Amostragem domiciliar continua em estudos epidemiológicos e no ensino. Rev. Saúde públ., S. Paulo, 13; $195-202,1979$

RESUmo: Descreve-se um sistema continuo de levantamento de condiçoes de saúde, por entrevistas domiciliárias, operando em Ribeirāo Preto (SP) desde 1974. Comentam-se as vantagens auanto à sua utilização na investigação de problemas especificos surgidos nesse periodo, bem como a sua utilização no ensino.

UnITERMos: Amostragem. Inquíritos sanitários. Epidemiologia.

\section{N T IO D U C ÃO}

Nenlluuma investigação epidemiológica é melhor do que a qualidade das suas fontes de dados. Não bastassem as dificuldades inerentes aos sistemas de registro de dados no setor saude, acrescente-se a quase impossibilidade de obtenção das bases populacionais quando se trabalha a nivel regional ou mesmo municipal.

Raros são os estudos que levam às últimas consequiências uma análise a respeito $\mathrm{da}$ "área de influência" de um hospital ou de qualquer outro serviço de saúde. Num sistema de atenção médica não regionalizado torna-se impossivel, de um lado, estabelecer barreiras ao acesso da população aos serviços de saúde e, de outro, utilizar os registros rotineiros como boa fonte de informação epidemiológica. Uma das maneiras de contornar o problema é a realização de investigações epidemiológicas na comunidade, centrando a análise em conjuntos especiais de pessoas nas quais se possa evidenciar a presença dos fatores suspeitos e de seus possiveis efeitos (a doença).

Desta forma consegue-se a necessária base populacional, indispensável a qualquer estudo epidemiológico. A solução implica forçosamente o emprego de técnicas de amostragem, já que a alternativa seria a modificação não apenas do sistema de re-

* Trabalho apresentado no III Simpósio Nacional de Probabilidade e Estatística, 1978.

* Do Departamento de Medicina Social da Faculdade de Medicina de Ribeirảo Preto Preto da USP - "Campus" de Ribeirão Preto - 14100 - Ribeirão Preto, SP - Brasil.

*** Do Departamento de Enfermagem Geral e Especializada da Escola de Enfermagem de Ribeirão Preto da USP - "Campus" de Ribeirão Preto - 14100 - Ribeirão Preto. SP - Brasil. 
CARVALHEIRO, J. da R. \& SANCHES, O. Amostragem domiciliar contínua em estudos epidemiológicos e no ensino. Rev. Saúde públ.. S. Paulo. 13:195-202. 1979.

gistro de dados mas, tambén, do próprio sistema de atenção à saúde.

Um esquema de levantamento de condiçōes de saúde por amostragem domiciliar trará inúmeras vantagens, desde que se lhe dê um caráter de continuidade. Em primeiro lugar, permitirá o fornecimento de base populacional às investigações epidemiológicas que se deseje implementar. Em segundo, uma análise crítica contínua do desempenho do próprio sistema de saúde ("monitorização") pelo cotejo da "demanda satisfeita" com a "morbidade percebida" e a "demanda expressa", avaliadas todas ao nivel da própria comunidade. Em terceiro, a sua utilização como fonte de dados e campo de treinamento em cursos, de graduação ou pós-graduação, de epidemiologia e/ou amostragem.

No presente trabalho é feita a descriçáo de um sistema contínuo de levantamento de condições de saúde, por entrevistas domiciliárias, operando em Ribeirão Preto (SP) desde 1974. Comentam-se as vantagens quanto à sua utilização na investigação de problemas específicos surgidos nesse periodo, bem como a sua utilização no ensino.

\section{LEVANTAMENTO DE CONDIÇOES DE SAÚDE POR ENTREVISTAS DOMICILIARIAS}

Realizaram-se duas experiências preliminares, desenvolvidas em 1972 (na Vila Guatapará) e 1973 (Vila Lobato), a primeira sede de um distrito e a segunda um bairro da cidade de Ribeirão Preto. A partir dos resultados dessas experiências, planejou-se um levantamento capaz de cobrir toda a área urbana da cidade de Ribeirāo Preto (Carvalheiro ${ }^{3}, 1975$ ).

Imaginou-se, preliminarnente, a definição de uma "amostra mestra", da qual pudessem ser extraidas sub-amostras para a realização de investigações específicas, esquema similar ao empregado por Holland e sua equipe do Hospital S. Thomas, no bairro londrino de Lambeth North e cujas bases estão descritas na literatura (citado por Bennett e Kasap", 1970). Optou-se, posteriormente, por um delineamento em que os bairros da cidade pudessem participar com pesos proporcionais ao tamanho de suas populações no censo de 1970. Como base para este delineamento adotou-se esquema de amostragem scmelhante ao empregado pelo "National Center for Health Statistics"s, pelo IBGE na sua Pesquisa Nacional por Amostra de Domicilios $(P N A D)^{1}$ e pela "Direccion General de Estadistica de Mexico".

Embora nunca se tenha pretendido atingir o nivel de sofisticação dessas investigações conduzidas, a nivel nacional, por organismos especializados na execução de censos, procurou-se seguir uma metodologia similar que viesse facilitar uma posterior expansão da área de levantamento, pelo menos até os limites de VI Região Administrativa do Estado de São Paulo.

$O$ delineamento da amostragem conduz a uma amostra probabilística, de estágos mútiplos e estratificada, da população civil não institucionalizada da cidade de Ribeirāo Preto. Para consegui-la partiu-se da divisão da cidade em setores censitários realizada pelo IBGE para a execução do censo de 1970. Uma cópia do mapa da cidade mostrando essa divisão, bem como das folhas de coleta de onde se podia obter a população residente em cada um desses setores, distribuida por sexo, foram obtidas na Secretaria de Planejamento do Municipio de Ribeirão Preto. Era possivel, desta forma, recompor a subdivisão da cidade em bairros e a composição destes em setores pela comparação desse material com a tabela de distribuição da população da cidade pelos bairros, fornecida pelo IBGE à VI Divisão Regional de Saúde. Verificou-se que a cidade fora dividida pelo IBGE en 175 setores para o censo de 1970 . Excluidos alguns setores, considerados áreas especiais pelo IBGE (constituidos por hospitais, asilos, cadeia, quartéis, colégios, hotéis, seminários, cemitérios), em número de 28, os demais eram compostos por um 
CARVALHEIRO, J. da R. \& SANCHES, O. Amostragem domiciliar contínua em estudos epidemiológicos e no ensino. Rev. Saúde públ., S. Paulo, 13:195-202, 1979.

número variável de quadras mas com un número aproximadamente constante de casas $(250$ a 300$)$. Os setores centrais, mais densos, compunham-se de poucas quadras, o inverso ocorrendo com os periféricos. Em vista dos recursos humanos e materiais disponiveis decidiu-se que poderiam figurar na amostra semanal $1 \%$ dos domicílios da cidade de acordo com os resultados do censo de 1970 , i.e., 380 domicílios. Como se pretendia um levantamento continuo decidiu-se partir de um painel de amostragem 7 vezes maior $e$ composto de cerca de 2.500 casas, distribuidas por aproximadamente um décimo dos setores do IBGE. Restou assim o problema de como distribuir esses 18 setores pelos bairros da cidade de Ribeirão Preto. Seguindo o procedimento usual nesse tipo de amostragem convencionou-se chamar bairros auto-representativos (BAR) aqueles cuja população fosse igual ou maior que 10 mil habitantes, resultado aproximado da divisão da população residente em 1970 na cidade de Ribeirão Preto (190.955) pelo número de setores que se pretendia sortear (18). Os demais bairros foram considerados não auto-representativos (BNAR) e agrupados em 3 estratos (alto, médio e baixo) de acordo com suas características. As caracteristicas consideradas foram as benfeitorias públicas existentes (água, luz, esgoto, iluminação pública, telefone, pavimentação das ruas) bem como as características dominantes das residèncias. Desta forma os BAR participaram obrigatoriamente da amostra com um número de setores proporcional à sua população no censo de 1970 , e os BNAR foram agrupados em 3 estratos sendo cada um destes incluído na amostra com um número de setores proporcional à sua população em 1970 .

Uma vez deterninado o número de setores a ser incluido na amostra em cada BAR e em cada estrato de BNAR tratou-se de sortear esses setores. Isso foi feito de tal maneira que a probabilidade de cada setor ser incluido na amostra fosse proporcional à sua população no censo de 1970 .
Tendo sido sorteados os 18 setores que figurariam na amostra foram todos desenhados em detalhe, com indicação do número de casas encontrado, no censo de 1970, em cada face de quadra. Obtidos estes mapas detalhados foram os setores subdivididos em áreas compostas de aproximadamente 60 casas. Cada setor ficou assim subdividido em um número variável de áreas que nunca foi inferior a 4 , nem superior a 6 . Havia sido estabelecido que cada setor figuraria na amostra com 2 destas áreas, que foram esscolhidas aleatoriamente com probabilidade proporcional às suas populações em 1970.

Iniciou-se então o processo de listagem para o qual se elaboraram vários formulários. $\mathrm{O}$ primeiro deles corresponde à Caderneta do Recenseador do IBGE, extremamente simplificada. $\mathrm{Na}$ folha de face consta apenas a identificação da área de listagem e a maneira mais fácil de atingi-la. $\mathrm{Na}$ parte interna figura um mapa detalhado da área, incluindo a localização das casas em cada quadra. Os entrevistadores, no campo, deveriam atualizar estes mapas, entregar em cada domicílio uma carta de apresentação e pedido de colaboração e proceder à listagem seguindo as instruções contidas no manual correspondente.

Terminada a listagem, iniciou-se o levantamento propriamente dito. O trabalho de campo passou a ser desenvolvido semanalmente, utilizando entrevistadores especialmente treinados para o preenchimento de formulários específicos. Nesses formulários investigava-se a ocorrência, na quinzena que precede a semana de entrevista, de episódios de doença, acidentes e utilização de serviços de saúde.

O trabalho foi desenvolvido, com algumas interrupções, até o final de 1976. Paralisado durante 1977 foi retomado em 1978, obrigando a uma atualização do painel de amostragem. 
CARVAlHeiro, J. da R. \& SANCHES, O. Amostragem domiciliar continua em estudos epidemiológicos e no ensino. Rev. Saúde públ. S. Paulo, 13:195-202, 1979.

T A B E L A 1

Bairros de Riberrảo Preto com indicação de suas populaçóes residentes no censo de 1970 (IBGE) e indicação do número de setores incluidos na amostra ( $K$ ) e o número de casas risitadas semanalmente em cada um deles.

\begin{tabular}{|c|c|c|c|c|}
\hline Categoria & Bairro & População & $\mathrm{K}$ & $\begin{array}{l}\text { Casas na } \\
\text { amostra }\end{array}$ \\
\hline \multirow{8}{*}{$\begin{array}{l}\text { Bairros auto- } \\
\text {-representativos } \\
\text { (BAR) }\end{array}$} & Centro & 19688 & 2 & $: 36$ \\
\hline & Jardim Paulısta & $1: 9.8 x$ & 1 & 28 \\
\hline & Vila Virgrina & $2025: 3$ & $\ddot{z}$ & 36 \\
\hline & Ipiranga & 18138 & 2 & 36 \\
\hline & Vila Recreio & 11083 & 1 & 201 \\
\hline & Vila Tibério & 18952 & 2 & 40 \\
\hline & Campos Elíseos & 30219 & 3 & 66 \\
\hline & Lapa & 11986 & 1 & 24 \\
\hline Total (BAR) & & $144 \quad 257$ & 14 & $28 b$ \\
\hline \multirow{4}{*}{ BNAR (Alta) } & $\begin{array}{l}\text { Sumaré } \\
\text { Higienópolis }\end{array}$ & $\begin{array}{l}14014 \\
618\end{array}$ & - & 18 \\
\hline & Jardim Universitário & 380 & $\cdots$ & \\
\hline & Jardim Irajá & 1160 & - & \\
\hline & Trital & (1) 127 & 1 & 18 \\
\hline \multirow{7}{*}{ BNAR (Média) } & Vila Lobato & 9314 & $*$ & 26 \\
\hline & Vila Tamandaré & 6251 & - & \\
\hline & Vila Seixas & $6: 303$ & - & \\
\hline & Faculdade de Medicina & 562 & - & \\
\hline & Jardim Independência & 1790 & + & 26 \\
\hline & Santa Terezinha & 1366 & $-\cdots$ & \\
\hline & Total & 25586 & 2 & 52 \\
\hline \multirow{7}{*}{ BNAR (Baixa) } & Santa Cruz & 1658 & - & \\
\hline & Vila Branca & 1790 & - & \\
\hline & Vila Abranches & 1098 & $\theta$ & 24 \\
\hline & Monte Alegre & 1019 & - & \\
\hline & Vila Carralho & 4898 & - & \\
\hline & Jardim Irajá & 1522 & - & \\
\hline & Total & 11985 & 1 & 24 \\
\hline \multicolumn{2}{|l|}{ Total (BNAR) } & 46698 & 4 & 94 \\
\hline \multicolumn{2}{|l|}{ Total geral } & 190955 & 18 & 380 \\
\hline
\end{tabular}

* BNAR sorteado para representar o seu estrato. 
CARVALHEIRO, J. da R. \& SANCHES, O. Amostragem domiciliar contínua em estudos epidemiológicos e no ensino. Rev. Saúde públ., S. Paulo, 13:195-202, 1979.

O primeiro painel era composto de 2.520 domicilios dos quais, semanalmente, se entrevistavam 380 . A repetição das visitas ao mesmo domicílio, com os inconvenientes dai decorrentes, forçaram inicialmente a uma ampliação do painel para 5.170 domicilios e, a seguir, a um maior espaçamento das visitas através da realização quinzenal do levantamento.
A atualização, em 1978, permitiu a inclusão de novos bairros e áreas de crescimento acelerado o que elevou para 8.431 os domicílios componentes do painel.

Nesse período foram realizadas 20.327 entrevistas, preenchidos 29.768 formulários de morbidade e acidente, 3.011 formulários de utilização de serviços de saúde e 1.626 de consumo de medicamentos.

T A B E L A

$$
\text { Produçăo global do sistema de entrevistas domicilárias sobre condições de saúde, }
$$
Ribeirão Preto, 1974-1978.

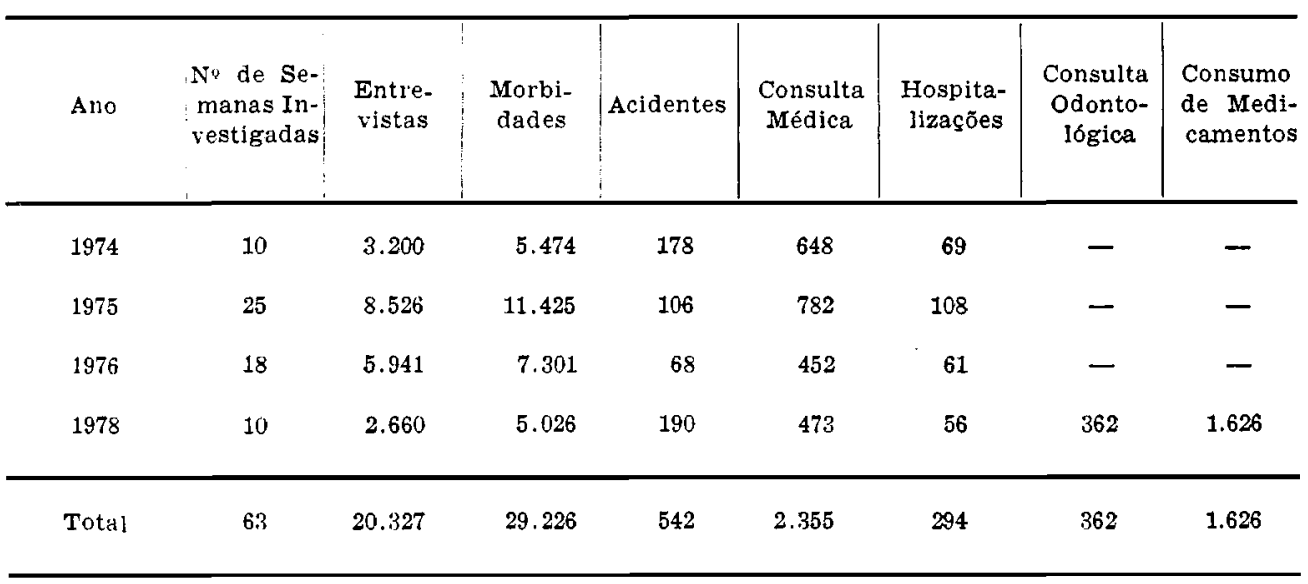

\section{REALIZAÇ.ÃO DE INVESTIGAÇŐES EPIDEMIOLOGICAS}

Puderam ser incluidas no trabalho rotineiro algumas investigações especificas, utilizando a mesma amostra. Para tanto foi suficiente a elaboração do formulário capaz de dar resposta às indagações implicitas nessas investigações, proceder à sua validação, treinamento das entrevistadoras $e$, finalmente, inclui-lo no elenco de formulários a serem preenchidos. Assim se procedeu para o levantamento da cobertura da campanha de vacinação anti-meningocócica, cobertura dos programas de vacinação em geral, consumo de medicamentos, referência a fenômenos inusitados durante a audiência a um programa de televisão (Uri Geller).

A realização de investigações epidemiológicas mais precisas esbarra com um problema fundamental: a definição operacional da "doença" investigada. Se quizermos fugir da mera definição de "morbidade sentida", deveremos lançar mão de instrumentos mais adequados. Estão propostos, em vias de implementação, estudos empregando questionários padronizados, aplicados pelas próprias entrevistadoras leigas e outros que implicam a realização de exames clínicos e coleta de material a nível dos domicílios. 
CARVALHEIRO, J. da R. \& SANCHES. O. Amostragem domiciliar contínua em estudos epidemiológicos e no ensino. Rer. Saúde públ., S. Paulo, 13:195-202, 1979.

\section{AVALIACAO DO DESEMPENHO DO SISTEMA DE SAÚDE}

Embora não se possam considerar como definitivos, os resultados até aqui obtidos nos animam a considerar o procedimento como capaz de fornecer condições ao desenvolvimento de projetos que não se prendam ao exclusivismo dos dados obtidos ao nivel do sistema formal de saúde. Admitindo que, ainda que confiáveis, estes dados estão sempre impregnados da visão oficial do papel e, portanto, do feitio dos serviços de saúde, é fácil avaliar a potencialidade do método no sentido de avaliar, criticamente, o desempenho do sistema de saúde.

\section{UTILIZAÇĀo NO ENSINO DE EPIDEMIOLOGIA E AMOSTRAGEM}

\section{Ensino ao nivel de graduação}

O Painel de Amostragem tem funcionado como uma fonte de dados atuais para ilustraçāo ou levantamento de problemas concretos de saúde, nos cursos de graduação da área de medicina preventiva, especialmente os de ciências sociais aplicadas, demografia e epidemiologia.

\section{Ensino ao nivel de pós-graduação}

$\mathrm{Na}$ área de medicina preventiva, ao nivel de pós-graduação, foi oferecido curso de "Métodos de Inquérito em Investigação Epidemiológica".

$\mathrm{Na}$ área de Bioestatística, quase que simultaneamente, foi oferecido um curso de "Amostragem".

O primeiro tem, entre outros, os objetiros de:

a) pôr em contacto os alunos de pós-graduação, da área de medicina preventiva, com o procedimento geral de amostragen utilizado no Painel à que já nos referimos;

b) reunir os estudantes en grupos com a finalidade de elaborar e desenvolver projetos específicos de investigação, os quais, tanto quanto possível, se utilizan do Painel de Amostragem.

A disciplina de Amostragem, da área de Bioestatistica, era oferecida exclusivamente aos alunos desta área, e ministrada de maneira formal, com base nos livros textos comumente indicados.

A partir de 1978 , no primeiro semestre, a disciplina foj oferecida também aos pós-graduandos da área de medicina preventira, enquanto que a disciplina de "Métodos de Inquéritos em Investigação Epidemiológica" oferecia possibilidade de matricula aos estudantes da Bioestatistica que estivessem cursando a disciplina de Amostragem.

Esta foi uma experiência didática que objetivava:

a) dar conhecimento basico, mais especifico, em técnicas de amostragem, ao pós-graduando da área de medicina preventiva;

b) dar, ao pós-graduando da área de bjoestatística, nāo apenas o conhecimento teórico da amostragem, mas permitir e incentivar a participação do mesmo na elaboração de projetos específicos de inrestigação que viessem a se utilizar de técnicas de amostragem.

Assim, os estudantes da área da bioestatística foram distribuidos dentro dos vários grupos de investigação formados na disciplina de "Inquéritos", com a obrigação de participar desde o planejamento da investigação e elaboração das hipóteses, até a análise final dos dados, passando pelas fases intermediárias de solução dos problemas administrativos e de campo. Foram preparados pelos alunos do curso um total de 4 projetos, além de um trabalho de apreciação geral do sistema de amostragem empregado. Desses projetos 2 utilizavam 0 painel de amostragem do sistema: "Influência da lei de vacinação na cobertura de vacinaçāo de menores de doze anos en Ribeirão Preto" e "Estudo de prevalência de doenças respiratórias crônicas obstrutivas mediante entrevistas domiciliárias". Os outros 2, utilizaram painéis de amostragem 
CARVAlHEIRO, J. da R. \& SANCHES, O. Amostragem domiciliar continua em estudos epidemiológicos e no ensino. Rev. Saúde públ., S. Paulo, 13:195-202, 1979.

diferentes: "Estudos de tentativas de suicídio na cidade de Ribeirão Preto" e "Influência do mercado de trabalho sobre a atuação do médico".

Este tipo de experiência didática foi montada, pela primeira vez no campus de Ribeirão Preto, por estarmos convencidos que mesmo o treinamento em assessoria estatística, através dos chamados Laboratórios de Estatística, não dá, ao pós-graduando em Bioestatística, a visão e a vivência necessárias para a solução de problemas específicos de amostragem, principalmente quando estão envolvidas populações humanas. Aliás, nunca é demais citar Yates ${ }^{6}$ (1965):

"We cannot devise good statistical methods merely by sitting in our studies and theorizing. Good statistical methods almost invariably result from contact between the mathematical statistician and the workers who are interested not in the statistical methods themselves, but in the conclusions that emerge from their work. Consequently, the statistician must be in intimate contact with the actual numerical data".

É claro que, com esta experiência, tentávamos um passo a mais: não só o con- tacto com os dados, mas o contacto com o problema, na sua essência e na sua origem.

A associação do Painel às disciplinas de "Métodos de Inquéritos em Investigação Epidemiológica" e "Amostragem" permitiu, aos estudantes de ambas as áreas, um contacto direto com problemas reais de investigação na comunidade, tendo culminado com a elaboração de projetos específicos de pesquisa.

Especialmente com relação aos estudantes da área da bioestatística, oriundos de cursos de graduação em matemática e estatística, esta experiência mostrou-se vantajosa no sentido que:

a) os mesmos começam a ter contacto com outra linguagem, que não é matemática, mas com a qual deverão estar familiarizados para exercer suas atividades profissionais no campo da saúde;

b) tomam contacto com bibliografias específicas, em amostragem, que transcendem os livros textos usados nos cursos formais. Isto principia por criar, nos mesmos, atitudes de análise crítica, que tendem a se aprimorar com as reunióes conjuntas de grupos para discussão dos projetos específicos.

RSPUB9/463

CARVAlHEIRO, J. DA R. \& SANChes O. IContinuous household sampling for epidemiological research and for teaching purposes/ Rev. Saúde púbi., S. Paulo, 13: $195-202,1979$.

ABSTRACT: The use of adequate populational-base survey is frequently impossible in epidemiological studies. Special studies are made among particular groups of individuals to investigate simultaneously the presence of both the factor and the disease. In these studies it is obviously important to use adequate sampling techniques. A system of continuous household sampling is described, designed to perform, simultaneously, epidemiological research, health system monitoring and to serve as a basis for courses on sampling techniques and epidemiological methods. In the municipality of Ribeirão Preto, S. Paulo, Brazil a household sampling system has been in operation since 1974, using a master sample of 8500 households. Every two weeks, 380 households are visited and information is gathered about diseases, accidents, and the use of health services. Special epidemiological research is introduced when necessary. Future development includes the use of standardized questionnaires and physical and laboratory examinations of the people interviewed.

UnITERms: Sampling studies. Health surveys. Epidemiology. 
CARVAlHEIRo, J. da R. \& SANCHES, O. Amostragem domiciliar contínua em estudos epidemiológicos e no ensino. Rev. Saúde públ., S. Paulo, 13:195-202, 1979.

\section{REFERENCIAS BIBLIOGRAFICAS}

1. BARBOSA, C. M. \& IINDQUIST, M. V. Programa de pesquisas domiciliárias no Brasil. Rev. bras. Estat., 32:298$330,1971$.

2. BENNETT, A. E. \& KASAP, H. S. Data processing for a private census. In: Holland, W. W., ed. Data hanlling in epidemiology. London, Oxford University Press, 1970. p. 111-23.

3. CARVALHEIRO, J. R. Levantamento de condicões de saude por entrevistas domiciliárias. Ribeirão Preto, 1975. [Tese de Livre-Docencia - Faculdade de Medicina de Ribeirão Preto da USP]
4. GARCIA, N. J. Diseño de la muestra de la encuesta nacional de hogares. Mexico, Dirección General de Estadistica, 1974.

5. NATIONAL HEALTH SURVEY. The statistical design of the health household: interview survey. Washington, 1958 (Health Statistics. Series A-2)

6. YATES, F. Sampling methods for censuses and surveys. 3rd ed. London. Charles Griffin, 1965.

Recebido para publicação em 22/03/1979

Aprovado para publicação em 19/06/1979 